\title{
Double coherence resonance in neuron models driven by discrete correlated noise
}

\author{
Thomas Kreuz,, , $*$ Stefano Luccioli, ${ }^{1,2}$ and Alessandro Torcini ${ }^{1,2}$ \\ ${ }^{1}$ Istituto dei Sistemi Complessi - CNR, Sesto Fiorentino, Italy \\ ${ }^{2}$ Istituto Nazionale di Fisica Nucleare, Unitá di Firenze, Sesto Fiorentino, Italy
}

(Dated: April 27, 2018)

\begin{abstract}
We study the influence of correlations among discrete stochastic excitatory or inhibitory inputs on the response of the FitzHugh-Nagumo neuron model. For any level of correlation the emitted signal exhibits at some finite noise intensity a maximal degree of regularity, i.e., a coherence resonance. Furthermore, for either inhibitory or excitatory correlated stimuli a Double Coherence Resonance (DCR) is observable. DCR refers to a (absolute) maximum coherence in the output occurring for an optimal combination of noise variance and correlation. All these effects can be explained by taking advantage of the discrete nature of the correlated inputs.

PACS numbers: 87.19.La,02.50.Fz,05.40.-a,87.10.+e
\end{abstract}

Excitable systems driven by fluctuations exhibit a large variety of phenomena where noise plays a constructive role. Among the most studied are stochastic resonance, the enhanced detectability of weak periodic stimuli for an intermediate noise level [1], and coherence resonance $(\mathrm{CR})$, the regularization of the system response at an optimal noise intensity without any external drive [2, 3]. $\mathrm{CR}$ has been observed in many theoretical and experimental setups such as electronic devices, semiconductor lasers, and climate models (for a recent review cf. [4]). In neuroscience evidence of $\mathrm{CR}$ has been reported for the cat's spino-cortical activity [5] as well as for a variety of single neuronal models. A prominent example is the two-dimensional FitzHugh-Nagumo (FHN) model [ [ ] that incorporates essential neuronal properties such as threshold dynamics (activation) and refractoriness.

A second type of $\mathrm{CR}$ with respect to the level of correlation has been observed in research areas as diverse as laser dynamics [7], digital circuits [8], chemical reactions [9] and neuronal models [10]. In computational neuroscience continuous noise is replaced by a series of discrete random kicks representing the post-synaptic potentials (PSPs) released by excitatory and inhibitory synapses [11, 12, 13, 14, 15]. Correlations are then introduced via the method of shared input (cf. [11, 12, 13]) such that the correlation equals the probability that different synapses deliver PSPs at the same time. Whereas the classical CR with respect to the noise intensity can be explained by the different dependencies of slow activation and fast excitation on the noise variance [3, 16, 17] so far the origin of the second type of CR has remained unclear.

In this Letter we address this issue by investigating a FHN model driven by a large number of stochastic synaptic inputs with correlations only among trains of either excitatory or inhibitory PSPs. In both cases the system exhibits the classical CR at any level of correlation, whereas for almost all noise strengths the second type of $\mathrm{CR}$ can be observed. The discrete nature of the inputs allows to vary independently correlation strength and noise intensity and thus to disclose the different mechanisms responsible not only for the CRs but also for the Double Coherence Resonance (DCR, i.e., the existence of an optimal combination of noise and correlation strength for which the system responds with maximal coherence) discovered for both excitatory and inhibitory correlation.

The FHN model can be written as

$$
\dot{V}=\phi\left(V-\frac{V^{3}}{3}-W\right) \quad, \quad \dot{W}=V+a-I(t),
$$

with a voltage variable $V$, a recovery variable $W$ and $I(t)$ the external synaptic input. We set time scale separation $\phi=100$ and bifurcation parameter $a=a_{0} \equiv 1.05$ so that the dynamics has only one attractor (a stable focus) but is close to the supercritical Hopf bifurcation at $a=1$.

In this study we consider a balanced FHN neuron in the high-input regime [1] with the input modelled as the superposition of an equal number $N \sim 100-10000$ of Poissonian trains of excitatory and inhibitory PSPs (EPSPs and IPSPs, respectively) with the same rate $\nu_{0}$

$$
I(t)=\Delta W_{0}\left[\sum_{i=1}^{N} \sum_{j} \delta\left(t-t_{i}^{j}\right)-\sum_{k=1}^{N} \sum_{l} \delta\left(t-t_{k}^{l}\right)\right]
$$

where $t_{i}^{j}\left(t_{k}^{l}\right)$ are the times of the instantaneous excitatory (inhibitory) kicks of amplitude $\Delta W_{0}$. The neuron fires upon a single excitatory kick with amplitude higher than $(\Delta W)_{c} \simeq 0.0138$ (for $\left.a=a_{0}\right)$. However, here we consider kicks of much smaller amplitude $\Delta W_{0}=0.0014$ with rates $\nu_{0}=0.3,0.6$, and 1.2 comparable with the firing rate $\nu_{f}=\nu_{f}(a)$ just beyond the bifurcation [21].

We examine the influence of input correlation $\rho_{x}$ on the neuronal response restricting ourselves to correlation among either excitatory $(x=e)$ or inhibitory $(x=i)$ inputs only. Correlations are expressed in terms of the Pearson correlation coefficient $\rho_{x}$ equal to the average fraction of shared kicks delivered by each pair of synapses or, analogously, to the average fraction of synapses delivering kicks at the same time [12]. Since in our model the inputs of different synapses are not distinguishable their effect can be reproduced by two over-all kick trains 
corresponding to correlated and uncorrelated PSPs, respectively. While the superposition of the uncorrelated kick trains can be modelled as a single Poissonian sequence of PSPs of constant amplitude $\Delta W_{0}$ and rates $\nu_{u}=N \nu_{0} \sim 5-1200$ quite high with respect to the natural firing frequency, correlated PSPs are generated using a refined method of shared inputs (cf. [11, 12, 13]). The superposition of the correlated kick trains (with correlation $\rho_{x}$ ) can be represented as a unique over-all Poissonian train of kicks of variable amplitude $\Delta W=n \times \Delta W_{0}$ and with constant rate $\nu_{x}=\nu_{0} / \rho_{x}$. The kick amplitude $n$ (in units of $\Delta W_{0}$ ) follows a binomial distribution

$$
p_{n}^{(N)}=\frac{N !}{n !(N-n) !} \rho_{x}^{n}\left(1-\rho_{x}\right)^{N-n}
$$

with average $\langle n\rangle=\rho_{x} N$ and variance $\operatorname{var}[n]=\rho_{x}(1-$ $\left.\rho_{x}\right) N$. For the balanced case the average input current is zero and does not depend on the correlation while the current variance per unit time $\sigma^{2}$ is determined by the variability of both correlated and uncorrelated kick trains: $\sigma^{2}=\Delta W_{0}^{2}\left[\langle n\rangle^{2}+\operatorname{var}[n]+\langle n\rangle\right] / T_{x}$ with $T_{x}=\nu_{x}^{-1}$.

For large $N$ the correlated kicks can be seen as large amplitude events that are delivered at a much lower rate $\nu_{x} \ll \nu_{u}$ than the uncorrelated inputs that can be assimilated to an almost continuous background. The effect of this background consists in renormalizing the bifurcation parameter according to $\bar{a}=a_{0} \pm\langle\Delta W\rangle / T_{x}$, the shift being positive (resp. negative) for $x=e$ (resp. $x=i$ ). The influence of the correlated kicks is embodied in the variance that for large $N$ (at the leading order) reads as $\sigma^{2} \simeq\langle\Delta W\rangle^{2} / T_{x}$. Thus in the high input regime the statistical properties of the response are determined once the average amplitude of the kick $\langle\Delta W\rangle$ and $T_{x}$ are known.

In the following we characterize the coherence of the neuronal response in dependence on noise strength $\sigma^{2}$ and correlation $\rho_{x}$ (or equivalently $T_{x}$ ) [22] changing the latter from full inhibitory correlation $\rho_{i}=1$ to full excitatory correlation $\rho_{e}=1$ including the completely uncorrelated case $\rho_{e}=\rho_{i}=0$. As indication of CR we employ the occurrence of a minimum in the coefficient of variation $R$ at intermediate noise levels. This quantity denotes the standard deviation of the distribution of the inter-spike time intervals (ISIs) normalized by its mean $\bar{T}_{I S I}$ and attains the value 0 for a perfectly regular signal and the value 1 for a Poissonian process.

For fixed $\rho_{x}$ the coefficient of variation $R$ exhibits a minimum for intermediate values of $\sigma^{2}$ in the whole range of excitatory and inhibitory correlations (cf. Fig. 1). Depending on the type of correlation we denote this effect as excitatory or inhibitory coherence resonance (eCR or iCR, respectively) 23]. Furthermore, by ordering the correlations from full inhibitory to full excitatory for almost all noise strengths $\sigma^{2}$ a minimum of $R$ can be observed at intermediate $\rho_{x}$. Finally, in each half-plane $\left(\sigma^{2}, \rho_{x}\right)$ (with $x=e$ and $x=i$, resp.) an overall minimum for

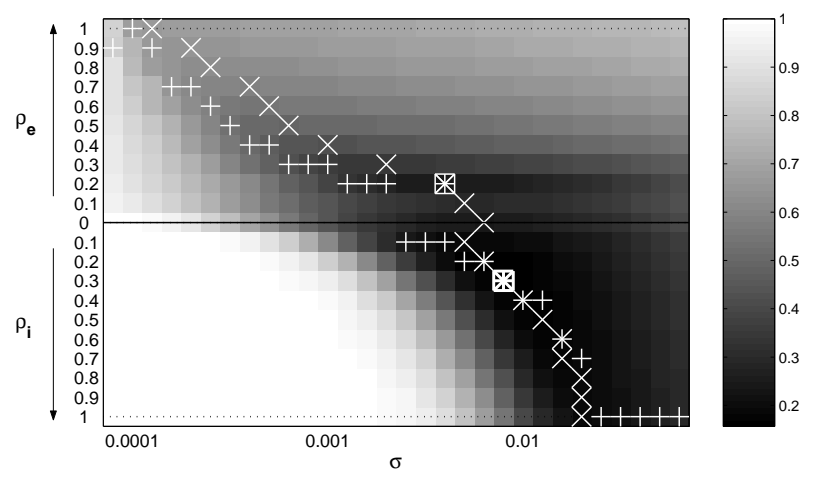

FIG. 1: Coefficient of variation $R$ as a function of noise strength $\sigma^{2}$ for both excitatory $\left(\rho_{e}\right)$ and inhibitory $\left(\rho_{i}\right)$ correlation. For the sake of brevity we project the axis of the two-dimensional plane $\left(\rho_{e}, \rho_{i}\right)$ on one single axis. In each column a white + marks the minimum for fixed variance, while a white $X$ in each row refers to the minimum for fixed correlation (i.e., the CRs). Horizontal lines mark the cases with no correlation, $\rho_{e}=1$ and $\rho_{i}=1$ exhibiting CR, eCR and iCR, respectively. Finally, the thin and thick white squares indicate the absolute minima obtained for excitatory and inhibitory correlation, i.e., the eDCR and iDCR, respectively. Data refer to $\nu_{0}=0.3$.

$R$ can be identified corresponding to excitatory and inhibitory Double Coherence Resonance (eDCR and iDCR, respectively). The iDCR is also the absolute minimum.

These effects can be better appreciated by considering the minimal value $R_{m}$ for each correlation as a function of $T_{x}$ (cf. Fig. 2). The curves for three different $\nu_{0}$ almost coincide indicating that once $T_{x}$ is fixed the minima $R_{m}$ occur at similar variances $\sigma_{m}^{2}$. Absolute minima are observed at finite $\rho_{x}$ and $\sigma^{2}$ both for excitatory (eDCR at $\bar{T}_{e} \equiv \bar{\rho}_{e} / \nu_{0} \simeq 0.65$ ) and inhibitory correlations (iDCR at $\left.\bar{T}_{i} \equiv \bar{\rho}_{i} / \nu_{0} \simeq 0.9\right)$. This behavior of $R_{m}$ is accompanied by a monotonous increase of the corresponding variances $\sigma_{m}^{2}$ when going from $\rho_{e}=1$ to $\rho_{i}=1$ (cf. Fig. 1). As we show in the following, not only $R_{m}$ and $\sigma_{m}^{2}$ depend on both type and strength of correlation, but also the underlying mechanisms are completely different from one another and in particular from the one responsible for CR without correlation [3].

Excitatory Coherence Resonance We begin with the origin of the $e C R$ at full excitatory correlation $\rho_{e}=1$. For this case the ISI-distribution of the output can be described, for any $\sigma^{2}$, in terms of a Poissonian process with average period $\bar{T}_{I S I}$ and refractory time $T_{\text {ref }}$. The expression of $R$ is simply given by $R_{P}=1-T_{\text {ref }} / \bar{T}_{I S I}$, however, the course of $R$ reveals a z-like shape with a clear minimum (the eCR, cf. inset of Fig. 2). At low variances firing resembles a noise activated process and therefore $\bar{T}_{I S I} \gg T_{\text {ref }}$ and $R=R_{P} \simeq 1$. For increasing $\sigma^{2}$ the average kick amplitude $\langle\Delta W\rangle$ gets larger until one single EPSP may be enough to trigger a spike, thus leading to more frequent firings and to an abrupt decrease of $R$. This quantity reaches its minimum when a $1: 1$ syn- 


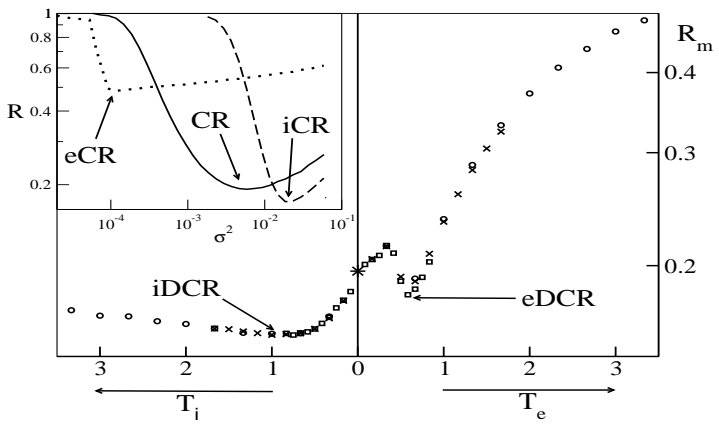

FIG. 2: Minimal values of the coefficient of variation $R_{m}$, obtained for fixed correlation, versus the period $T_{\rho}$. Data are ordered from full inhibitory correlation to full excitatory correlation. Symbols refer to different $\nu_{0}$-values: 0.3 (circles), 0.6 (crosses) and 1.2 (squares). The asterisk denotes the uncorrelated case and the arrows mark the positions of the double coherence resonances. Inset: $R$ versus $\sigma^{2}$ for different correlations $\left(\nu_{0}=0.3\right)$ : no correlation (solid line), $\rho_{e}=1$ (dotted line) and $\rho_{i}=1$ (dashed line). Arrows mark the position of the respective coherence resonances.

chronization between the EPSPs and the output spikes sets in since now each kick is sufficient to lead the system above the firing threshold from any state of the system (except during the refractory period). Accordingly, for $\sigma^{2} \geq \sigma_{m}^{2}$ the ISI-distribution exhibits a tail with slope $\nu_{e}$ and $R=R_{\text {sync }} \equiv 1-T_{\text {ref }} / T_{e}$. Further increasing $\langle\Delta W\rangle$ forces the system to fire even during the refractory period, thus leading to a reduction of $T_{\text {ref }}$ which explains the final growth of $R$ for $\sigma^{2}>\sigma_{m}^{2}$.

Excitatory Double Coherence Resonance This mechanism for eCR remains valid also for decreasing correlation $\rho_{e}$ until $R_{m}$ reaches its local minimum at $\bar{\rho}_{e}$ (corresponding to the eDCR, cf. Fig. 2). Beyond the eDCR, i.e., for $\rho_{e}<\bar{\rho}_{e}$, the $1: 1$ synchronization regime is no more reached (cf. 3k). For $\rho_{e}<\bar{\rho}_{e}$ the average kick amplitude $\langle\Delta W\rangle$ is always smaller than the minimal amplitude $(\Delta W)_{c}$ needed to elicit a spike (starting from the fixed point for the deterministic FHN, i.e., for Eq. 1 with $I(t) \equiv 0)$. For $\rho_{e} \geq \bar{\rho}_{e}$ and sufficiently high $\sigma^{2}$ (or analogously, $\bar{a}$ ) the average kick always overcomes the threshold $(\Delta W)_{c}$ thus confirming that the regime of $1: 1$ synchronization is always reached. Since the minimum of $R$ is associated with the onset of $1: 1$ synchronization (implying $\left.R_{m}=R_{\text {sync }}\right) R_{m}$ decreases for $1>\rho_{e}>\bar{\rho}_{e}$. For all correlations $\left[\bar{\rho}_{e} ; 1\right]$ this occurs roughly for the same amplitude $\langle\Delta W\rangle \simeq \Delta W_{c}$ (cf. Fig. 3 ), thus $T_{\text {ref }}$ is not significantly altered and the decrease of $R_{m}$ is due to the reduction of $T_{e}$ with decreasing correlation. Finally, beyond the $e D C R$, i.e., for $\rho_{e}<\bar{\rho}_{e}$, the system is no more strictly forced by the driving kick train with very high frequency, the period of firing decreases (i.e., $\bar{T}_{I S I}>T_{e}$ ) and thus $R_{m}$ increases.

Inhibitory Coherence Resonance Turning our attention to correlated inhibitory inputs we start with exam-
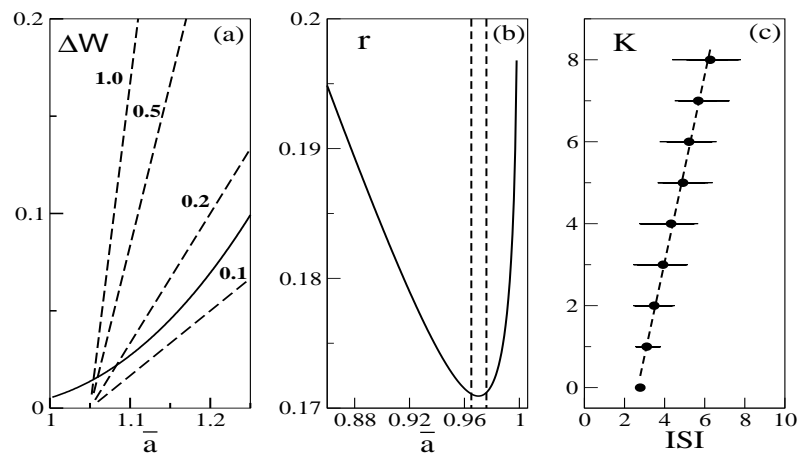

FIG. 3: Excitatory correlation: (a) Minimal amplitude $(\Delta W)_{c}$ (solid line) and average kick amplitude $\langle\Delta W\rangle$ (dashed lines) versus the renormalized bifurcation parameter $\bar{a}$. The number in proximity of the dashed lines indicate the corresponding values of $\rho_{e}$. Data refer to $\nu_{0}=0.3$, in this case $\bar{\rho}_{e} \sim 0.2$. Inhibitory correlation: (b) Ratio $r(\bar{a})$ for the deterministic FHN model, the values of $R_{m}$ for $\rho_{i}>\bar{\rho}_{i}$ are associated with $\bar{a}$-values located within the two dashed lines for al the examined $\nu_{0}$.(c) Number of inhibitory kicks $K$ received within a certain ISI as a function of its duration. Filled circles mark the average for each $K$. The dashed line indicates a linear fit, the inverse of its slope gives the delay per kick $\delta$. Data have been obtained from 6000 ISIs for $\nu_{0}=0.3, \rho_{i}=0.6, \sigma^{2}=0.06$.

ining the origin of the $i C R$ at full inhibitory correlation $\rho_{i}=1$. Once more at low variances the emission of spikes is due to an activation process with $R \sim 1$. For increasing $\sigma^{2}$ the renormalization of the bifurcation parameter, now due to the uncorrelated EPSPs, drives the system towards the repetitive firing regime eventually crossing the bifurcation at $\bar{a}=1$ and this leads to a fast decrease of $R$. On the other hand, the correlated IPSPs inhibit tonic firing, but despite the increase of their amplitude with $\sigma^{2}$ their action gets less effective. This is because the fraction of time $r(\bar{a})$ during which the neuron is sensitive to the arrival of a kick before a spike emission 24] decreases for increasing $\sigma^{2}$ (i.e., for smaller $\bar{a}$ ). Moreover, $r(\bar{a})$ exhibits a minimum at $\bar{a} \sim 0.97$. Noticeably, $R_{m}$ is associated with corresponding $\bar{a}$-values for all $\rho_{i}>\bar{\rho}_{i}$ (cf. Fig. 3b). Thus the minimum is due to the uncorrelated kick trains that renormalize the bifurcation parameter. The final increase of $R$ at large $\sigma^{2}>\sigma_{m}^{2}$ is reflected by a "quantization" in the ISI-distribution. The single ISI is proportional to the number of inhibitory kicks received within its duration (cf. Fig. 3r) and for high variances the delay per kick $\delta$, i.e., the average retardation (with respect to the natural firing period) of the next neuronal firing induced by a single inhibitory kick, increases and consequently the ISI-distribution broadens finally revealing a multimodal structure.

Inhibitory Double Coherence Resonance This mechanism for iCR is still valid for decreasing correlations $\rho_{i}$ until $R_{m}$ reaches its absolute minimum at $\bar{\rho}_{i}$ (corresponding to the $i D C R$, cf. Fig. 2). The decrease of $R_{m}$ with $\rho_{i}$ is due to the fact that $R_{m}$ is associated 
with an almost constant $\bar{a}$-value within the interval $\left[\bar{\rho}_{i} ; 1\right]$, and that for fixed $\bar{a}$ the amplitude of the inhibitory kick $\langle\Delta W\rangle \propto \rho_{i}$. Consequently the average delay per kick $\delta$ decreases with the correlation until the quantization in the ISI-distribution disappears at the iDCR at $\rho_{i}=\bar{\rho}_{i}$. For smaller $\rho_{i} \ll \bar{\rho}_{i}$ the frequency of the inhibitory kicks becomes more important than their amplitudes. In particular, lowering the correlation towards the uncorrelated case leads to an increasing rate $\nu_{i}$ of inhibitory disturbances that renders the neuronal spiking more irregular, which in turn is reflected by the increase of $R_{m}$. In summary, we described novel mechanisms for CR that are not, as in the uncorrelated case [3], related to the different nature of the stochastic processes underlying neuronal firing at low and high noise but that rely on the discrete nature of the correlated inputs. Furthermore, we reported the existence of DCRs both for excitatory and inhibitory correlations. These DCRs reflect the change from the classical CR 3] to amplitude-dominated mechanisms responsible for the $\mathrm{CR}$. While the eDCR is related to a complete synchronization with the input, the iDCR is due to a quantization of the neuronal output.

Our results indicate that the coherence in the response of an excitable system driven by fluctuations can be modulated by controlling independently the level of correlation and the noise variance. This could be of high relevance for neuronal coding since there are indications that correlated activities (as indeed measured, e.g., among cortical neurons [18]) can influence the coding ability of neuronal populations. More recently neuronal input correlation has been linked to changes in attention suggesting its major impact on the information flow in the brain [19]. Remarkably, in simulations with correlated inhibitory input it has been shown that attention (modeled as an increase of synchrony in interneuron networks) can lead to a decrease of the coefficient of variation of single output spike trains [20]. Furthermore, we have verified that the FHN with conductance-based inputs exhibits the same coherence effects as reported here for the currentdriven model. This analysis together with further extensions to more physiological setups will be the subject of future studies. Finally, since most of the reported results are not specifically related to the considered model we expect that analogous effects can be found also for excitable systems in other fields of research.

We acknowledge S. Lepri and A. Politi for useful dis- cussions and the European community for supporting TK via the Marie Curie IEF project No 011434.

* Electronic address: thomas.kreuz@fi.isc.cnr.it

[1] L. Gammaitoni et al., Rev Mod Phys 70 (1998) 223.

[2] Hu Gang et al., Phys Rev Lett 71 (1993) 807.

[3] A.S. Pikovsky and J. Kurths, Phys Rev Lett 78 (1997) 775.

[4] B. Lindner et al., Phys Rep 392 (2004) 321.

[5] E. Manjarrez et al., Neurosci Lett 326 (2002) 93.

[6] R. FitzHugh, Biophys J 1 (1961) 445.

[7] J.M. Buldú et al., Phys Rev E 64 (2001) 051109.

[8] S. Brugioni et al., Phys Rev E 71 (2005) 062101.

[9] V. Beato et al., Phys. Rev. E 71 (2005) 035204(R).

[10] J.M. Casado, Phys Lett A 235 (1997) 489.

[11] M.N. Shadlen and W.T. Newsome, J Neurosci 18 (1998) 3870.

[12] E. Salinas and T.J. Sejnowski, J Neurosci 20 (2000) 6193.

[13] M. Rudolph and A. Destexhe, Phys Rev Lett 86 (2001) 3662.

[14] J. Feng and P. Zhang, Phys Rev E, 63 (2001) 051902.

[15] R. Moreno et al., Phys Rev Lett 89 (2002) 288101.

[16] W.-J. Rappel and S.H. Strogatz, Phys Rev E 50 (1994) 3249.

[17] J.R. Pradines, G.V. Osipov, and J.J. Collins, Phys Rev E 60 (1999) 6407.

[18] E. Zohary, M.N. Shadlen, and W.T. Newsome, Nature 370 (1994) 140.

[19] E. Salinas and T.J. Sejnowski, Nature Rev Neurosci 2 (2001) 539.

[20] P.H.E. Tiesinga et al., J. Physiol. 98 (2004) 296.

[21] We use a fourth order Runge-Kutta integration scheme with time step $\delta t=10^{-4}$. A spike is identified whenever $W(t)$ overcomes a fixed detection threshold $\Theta=0.4$.

[22] First the correlation $\rho_{x}$ is chosen and then $\sigma^{2}$ is fixed (independently of $\rho_{x}$ ) by selecting the appropriate number of synapses $N$.

[23] The observed CR effects can not simply be due to the dependence of the output rate on the level of noise since for any fixed degree of correlation the rate increases strictly monotonously with the noise strength.

[24] This quantity can be evaluated for the deterministic FHN as the ratio $r(a)=1-T_{r e f}(a) / T_{f}(a)$, where $T_{f}(a)=$ $1 / \nu_{f}(a)$ is the period of tonic firing for Eq. (1) with $I \equiv 0$ and $T_{r e f}(a)$ the refractory period estimated as the time needed to recover after a spike emission from $(V, W)=$ $(1,2 / 3)$ to the fixed point. 